\title{
Age-related differences in memory after attending to distinctiveness or similarity during learning
}

\author{
Valerie A. Carr ${ }^{\mathrm{a}, \mathrm{b} *}$, Alan D. Castel $^{\mathrm{c}}$ and Barbara J. Knowlton ${ }^{\mathrm{c}}$ \\ ${ }^{a}$ Interdepartmental Program in Neuroscience, University of California, Los Angeles, CA, USA; \\ ${ }^{b}$ Department of Psychology, Stanford University, Stanford, CA, USA; ${ }^{c}$ Department of Psychology, \\ University of California, Los Angeles, CA, USA
}

(Received 14 August 2013; accepted 20 February 2014)

\begin{abstract}
Episodic memory is vulnerable to age-related change, with older adults demonstrating both impairments in retrieving contextual details and susceptibility to interference among similar events. Such impairments may be due in part to an age-related decline in the ability to encode distinct memory representations. Recent research has examined how manipulating stimulus properties to emphasize distinctiveness can reduce agerelated deficits in memory. However, few studies have addressed whether learning strategies that differentially encourage distinctiveness processing attenuate age-related differences in episodic memory. In the present study, participants engaged in two incidental encoding tasks emphasizing either distinctiveness or similarity processing. Results demonstrated higher rates of recollection for stimuli studied under the distinctiveness task than the similarity task in younger but not older adults. These findings suggest a declining capacity for distinctiveness processing to benefit memory in older adults, and raise the possibility that strategies that enhance gist-based encoding may attenuate age-related memory deficits.
\end{abstract}

Keywords: aging; memory; learning strategy; distinctiveness; recollection

Changes in memory across the life span have been well documented, providing evidence that episodic memory, or the capacity to encode and subsequently retrieve memories for events, is particularly vulnerable to age-related change (Mäntylä, 1993; McDaniel, Einstein, \& Jacoby, 2008; Old \& Naveh-Benjamin, 2008; Perfect, Williams, \& Anderton-Brown, 1995; Prull, Dawes, Martin, Rosenberg, \& Light, 2006). Successful episodic memory requires that memory representations be encoded distinctly, thereby allowing for retrieval of event-specific details and minimal interference across events. Given that older adults often incorrectly recognize novel stimuli as familiar, age-related impairments in episodic memory may be due in part to impairments in forming new memories that are sufficiently distinct from previously stored memories.

Support for this view comes from studies of spontaneous encoding processes that demonstrate that older adults tend to use less distinctive encoding processes than younger adults (Dodson \& Schacter, 2002; Mäntylä \& Bäckman, 1990; Rabinowitz, Craik, \& Ackerman, 1982). Such general, stereotyped encoding may lead to the formation of overlapping memory representations and, as such, may contribute to age-related deficits in episodic memory. Additionally, during tests of recognition memory, older adults have demonstrated impairments in discriminating targets from perceptually similar foils (Stark, Yassa, Lacy, \& Stark, 2013; Toner, Pirogovsky, Kirwan, \& Gilbert, 2009), suggesting an

*Corresponding author. Email: valerie.a.carr@gmail.com 
impaired ability to form distinct memory representations. The goal of the present study was to determine whether strategies that specifically orient attention to distinctiveness at encoding could attenuate age-related impairments in episodic memory.

Though little research has been conducted to address this question, a rich literature exists evaluating the effects on age-related differences in memory of manipulating stimulus properties to emphasize distinctiveness. For example, several studies have used the Deese-Roediger-McDermott (DRM) false recognition procedure (Deese, 1959; Roediger $\&$ McDermott, 1995) to evaluate memory performance in older and younger adults. Using this procedure, participants are more likely to falsely recognize lures that are semantically related to target items than those that are not. This effect can be attenuated in younger adults by presenting words visually; the interpretation is that if participants are generating semantically related lures during study, these will be more distinct from auditorily presented targets and thus easier to reject (Smith \& Hunt, 1998). The results have been mixed as to whether older adults show the same benefit of distinctiveness processing for rejecting semantically related lures (Schacter, Israel, \& Racine, 1999; Smith, Lozito, \& Bayen, 2005). However, there is some evidence that older adults can benefit from a distinctiveness heuristic at retrieval when given specific instructions to use distinctive information at test (Dodson \& Schacter, 2002). Furthermore, older adults show a reduction in false memory for visually compared to auditorily presented words when asked to recollect stimulus modality and to make judgments about the quality and characteristics of their memory for each item (Gallo \& Roediger, 2003). These results suggest that if sufficient environmental support is provided, older adults can use distinctiveness in a similar manner as younger adults to reduce false recognition.

Examining age-related differences in memory for distinctive items, Bruce and Herman (1986) found that younger adults better remember distinctive items than older adults, whereas no memory differences were found between age groups for typical items. Similarly, in a recent study by Badham, Wade, Watts, Woods, and Maylor (2013), the authors demonstrated that when participants try to identify a studied face in a simulated police lineup, younger adults are more accurate when distinctive facial features (e.g., scars or tattoos) are present in all members of the lineup compared to when they are deleted; in contrast, older adults show similar levels of accuracy when the distinctive features are present or absent from the faces in the lineup. Older adults also exhibit a reduced production effect, in that they show less improvement in memory than younger adults for words read aloud versus read silently (Lin \& MacLeod, 2012). As the benefit of reading words aloud has been attributed to increasing their distinctiveness, the reduced production effect in older adults may result from reduced distinctiveness processing. Taken together, these findings suggest that the benefits of distinctiveness to successful encoding decline with age.

A number of studies have also examined age differences in the isolation effect - that is, enhanced memory for items that differ from their context. In general, the isolation effect is either absent (Cimbalo \& Brink, 1982) or reduced to some extent (Bireta, Surprenant, \& Neath, 2008; Geraci, McDaniel, Manzano, \& Roediger, 2009) in older relative to younger adults (though see Badham \& Maylor, 2013, for evidence of isolation effects with equivalent magnitude among age groups). By one view, isolation effects depend on participants noticing the similarities among the nonisolates and how these differ from the isolated items (Hunt, 2006, 2013). Older adults may have a reduced ability to notice how isolates and nonisolates differ, and thus may have difficulties using these differences as effective retrieval cues at test. Supporting this view, research demonstrates that isolation effects in older adults can be boosted by facilitating the discovery of 
similarities between nonisolates. For example, older and younger adults showed equivalent isolation effects when the isolates were presented late in a list to allow similarities among the preceding items to become more apparent (Smith, 2011). Also, similar levels of the isolation effect were shown across age groups when the distinctive item was radically different from the other items, such as digits presented in a list of words (Smith, 2011). Furthermore, reduced age differences were shown in an examination of isolation effects based on font size in which younger adults reported greater spontaneous use of elaborative semantic encoding strategies than older adults (Vitali et al., 2006) strategies that may have attenuated the intended isolation effect in younger adults.

Taken together, these results suggest that while older adults may not necessarily remember distinctive items to the same degree as younger adults, under some circumstances age differences in memory can be reduced by manipulating stimulus properties or by enhancing environmental support for distinctiveness processing at retrieval. These results are consistent with the view that reduced cognitive resources, such as working memory efficiency, may impair the extent to which older adults spontaneously engage in distinctiveness processing (Smith, 2006). However, these studies do not address whether manipulating learning strategies to specifically encourage attention to distinctiveness improves memory - particularly episodic memory - in older adults. Given results that distinctiveness effects in memory can be improved in older adults by providing support for distinctiveness processing, it may be that explicitly directing attention to distinctive features could reduce age-related memory impairment.

It is well established that top-down processing during learning plays an important role in influencing what is encoded into memory (for reviews, see, e.g., Chun \& Turk-Browne, 2007; Craik, 2001). A recent functional magnetic resonance imaging study in young adults, for example, revealed that attending to distinctiveness at encoding resulted both in enhanced episodic memory performance and increased activity in the hippocampus - a region known to play a key role in episodic memory formation - relative to attending to similarities across stimuli (Carr, Engel, \& Knowlton, 2013). Two possibilities arise regarding the effects of such task demands on memory performance in older adults. The first is that recognition deficits among older adults may be attenuated by orienting participants away from strategies emphasizing generalities and toward those emphasizing distinctiveness. Similar to findings reported in studies of the isolation effect, facilitating the discovery of differences between stimuli may improve memory performance among older adults. Previous work has shown that instructing older adults to engage in deeper semantic processing leads to a qualitative reduction in memory impairments and significantly reduces prefrontal hypo-activation during encoding relative to younger adults (Logan, Sanders, Snyder, Morris, \& Buckner, 2002). From a mechanistic perspective, if older adults are explicitly oriented to distinctiveness at study, they may be able to better engage cortical regions thought to mediate goal-directed resources and in turn facilitate the formation of distinctive memory traces.

The second possibility arises from the view that the beneficial effects of distinctiveness on memory seen in younger adults are reduced in older adults due to a deficit in associating stimuli with their distinctive features (Naveh-Benjamin, 2000). By this view, older adults may not benefit from a learning strategy that orients attention to distinctiveness because of their difficulties with associating distinctive stimulus features with other features of the stimulus. In a similar vein, older adults may not benefit from orienting to distinctiveness if they have difficulty forming unique associative memories, as has been suggested by studies examining age-related changes in the hippocampus (Wilson, Gallagher, Eichenbaum, \& Tanila, 2006). 
Here, we directly addressed the question of whether and how encoding strategies that differentially emphasize distinctiveness processing influence recognition memory for faces in younger and older adults. Participants engaged in each of two incidental encoding tasks: one that directed participants' attention to distinctiveness, and one that required attention to similarity (modified from Mäntylä, 1997). In the distinctiveness task, participants are likely to focus on features that are unique to each face during encoding, whereas in the similarity task participants are likely evaluating multiple facial features to assess similarity across faces. Following encoding, participants performed a surprise recognition memory test assessing levels of recollection and familiarity (Tulving, 1985) for faces seen in both tasks. Using this type of test allowed us to assess whether, and how, distinctiveness processing differentially affects episodic memory as measured by recollection rates.

In a similar study conducted in young adults by Mäntylä (1997), results indicated that, compared to attending to similarities, attending to distinctiveness during incidental encoding selectively enhanced recollection in a recognition memory task. For this reason, we hypothesized that, for younger adults, overall recognition would not substantially differ for faces studied in the two tasks, but that they would show enhanced rates of recollection following the distinctiveness task relative to the similarity task. Conversely, based on data suggesting a reduced ability among older adults to associate stimuli with their distinctive stimulus features (Naveh-Benjamin, 2000) as well as a reduced ability to encode unique memory representations (Stark et al., 2013; Toner et al., 2009; Wilson et al., 2006), we hypothesized that recollection would not be modulated by encoding task in older participants. Given prior work demonstrating that older adults preferentially rely on gist-based memory (Brainerd, Reyna, \& Howe, 2009; Koutstaal \& Schacter, 1997), we also explored an alternative hypothesis that in older adults attending to similarity may lead to higher rates of recollection than attending to distinctiveness to the extent that processing similarity favors the formation of gist-based memories.

\section{Method \\ Participants}

Forty-seven undergraduate students (35 female), ranging from 18 to 30 years of age $(M=$ 20.55), were recruited from the University of California, Los Angeles (UCLA) campus to participate in the study. The older adult sample consisted of 24 healthy individuals (16 female) recruited from the greater Los Angeles community, ranging from 71 to 89 years of age $(M=80.13)$. All older participants had normal or corrected to normal hearing and eyesight, and scored 27 or higher on the Mini-mental State Examination (Folstein, Folstein, \& McHugh, 1975). Three older adults were excluded from the study due to inability to understand and/or follow task instructions. In return for participation in the study, students received course credit and older adults were paid $\$ 10$. The study was performed under a protocol approved by the UCLA Office for Protection of Research Participants.

\section{Materials}

All stimuli were presented via a Macintosh laptop computer (Apple, Inc., Cupertino, CA, USA) using the MATLAB (MathWorks, Natick, MA, USA) Psychophysics Toolbox. Stimuli consisted of 100 black and white photographs of 50 male and 50 female young adults, all of whom were Caucasian, with neutral facial expressions and a uniform background (see Figure 1; Psychological Image Collection at Stirling, http://pics.psych.stir.ac.uk). 
(a) Distinctiveness

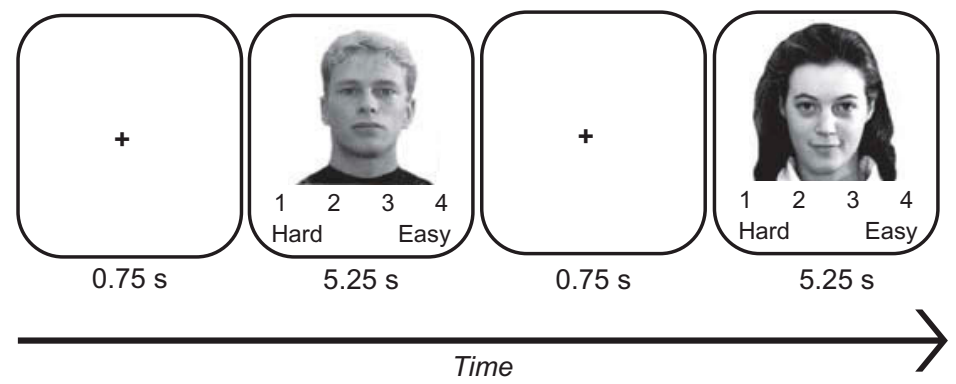

(b) Similarity

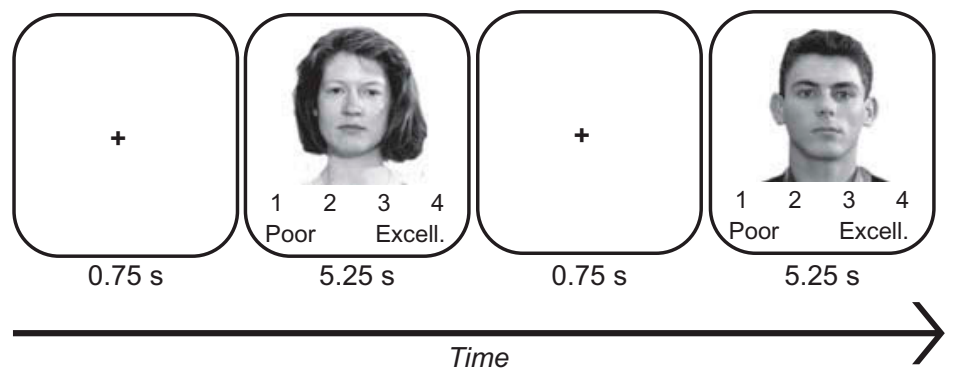

Figure 1. Distinctiveness and similarity task paradigms. During the distinctiveness task (a), participants imaged themselves as chauffeurs and rated how easily a given "customer" could be picked out of a crowded airport on a scale from 1 to 4 , ranging from $1=$ hard to $4=$ easy. During the similarity task (b), participants imagined themselves as casting agents and rated how well each "actor" matched the director's desired look on a scale from 1 to 4 , ranging from $1=$ poor match to $4=$ excellent match. Faces reproduced with permission from http://pics.psych.stir.ac.uk/.

Photographs included very little or no clothing, although hair color and style varied widely across participants. Faces were divided into four sets of 25 , such that 25 served as stimuli for the distinctiveness task, 25 for the similarity task, and the remaining two groups of 25 faces served as lures for the recognition test. Sets were counterbalanced across participants such that each set was assigned to the distinctiveness, similarity, or lure condition an equal number of times. Within a given task, stimulus presentation was randomized.

\section{Procedure}

Instructions to participants were identical to those reported in Carr et al. (2013) and are repeated here for completeness. For the distinctiveness, or "Taxi Driver" task (Figure 1a), participants were told to imagine that they were taxi drivers picking up specific customers from the airport. As each customer's photo appeared on the screen, participants made a subjective judgment regarding the ease with which they could find that particular customer in the crowd. This judgment was made on a scale from 1 to 4 ranging from $1=$ hard (customer has an average face and will blend in with crowd) to $4=$ easy (customer is very unique looking and will stand out in the crowd).

In the similarity, or "Casting Director" task (Figure 1b), participants were told to imagine themselves as casting directors who were auditioning actors for the lead male and female roles for a new movie. They were informed that the director desired a specific look 
for these roles, and that they should only audition actors matching this look. Participants were first shown a sample male and female actor fitting the desired look, and were then asked to rate a series of "head shots" according to how well each actor matched this overall look on a scale from $1-4$, ranging from $1=$ poor match to $4=$ excellent match.

The study employed a within-subject design such that all participants completed both encoding tasks, with task order counterbalanced across participants, prior to performing a surprise recognition task that assessed memory for faces from both tasks. Critically, participants were recruited to perform a study of "face processing," and thus were unaware of any memory-related aspects of the study design until they were asked to perform this recognition test. This procedure made it likely that participants used only those strategies introduced by the experimenters, whereas a procedure employing alternating study/test blocks would likely have introduced confounds between participants' intentional learning strategies and the incidental learning strategies intended by the experimenters.

The distinctiveness and similarity tasks were each composed of 25 trials, beginning with a brief fixation cross $(0.75 \mathrm{~s})$ followed by presentation of the face for $5.25 \mathrm{~s}$. Participants were asked to verbally rate each face according to task instructions within this $5.25 \mathrm{~s}$ period, with key-presses made by the experimenter. Following completion of the second encoding task, participants worked on a complex maze for $5 \mathrm{~min}$, which served as a distracter task to attenuate the possibility of ceiling and order effects on the surprise recognition test.

During the recognition test, participants viewed faces from each of the two encoding tasks (25 trials each) as well as 50 lure faces. For each face, participants made a two-step judgment (Eldridge, Sarfatti, \& Knowlton, 2002): a studied/new judgment followed by a remember/know judgment (Tulving, 1985) for those faces deemed studied. Participants were told to give a "remember" response when they remembered contextual details pertaining to when they originally viewed the face, and to give a "know" response when they confidently recognized a face in the absence of such details. This test was used to assess the extent to which successful recognition was based on episodic recollection or feelings of familiarity. To ensure that participants properly understood the distinction between remembering and knowing, they were asked to define each construct in their own words and to offer an example of each before beginning the recognition test.

Results from each participants' recognition test were used to sort trials into hits, misses, correct rejections, and false alarms; hits and false alarms were further broken down according to remember and know responses. To examine whether task demands at encoding influenced memory performance differently across age groups, we conducted a three-way mixed effects analysis of variance (ANOVA) examining hit rates in the two groups (younger and older adults) for the two encoding tasks (distinctiveness, similarity) broken down according to memory quality (remember, know). Given that the recognition test included faces from both tasks, false alarms were not specific to a given task, so corrected hit rates were not used. Planned comparisons were conducted using paired, twotailed $t$-tests (alpha level .05). A power analysis revealed that this sample size resulted in a probability of 0.8 to detect large pairwise effects (Cohen's $d>.7$ ).

\section{Results}

\section{Distinctiveness and similarity ratings}

Participants' response rates along each encoding task's respective four-point scale are shown in Figure 2. A mixed effects ANOVA evaluating the effects of age group (younger and older adults), encoding task (distinctiveness and similarity), and response option (1-4) 

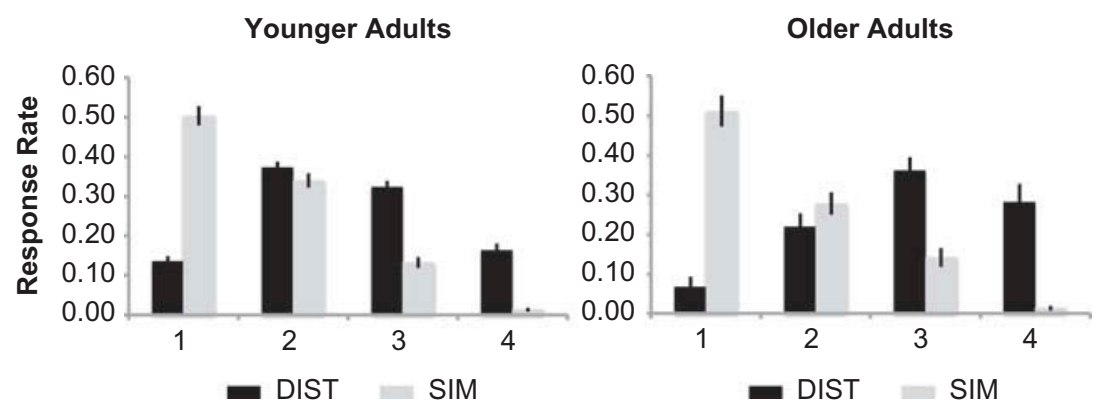

Figure 2. Encoding task ratings for each age group. In the distinctiveness task, ratings ranged from $1=$ hard to pick out of a crowd (not distinctive) to $4=$ easy to pick out of a crowd (very distinctive). For the similarity task, ratings ranged from $1=$ poor match (dissimilar) to $4=$ excellent match (very similar). Error bars represent standard error of the mean. DIST $=$ distinctiveness task, SIM = similarity task.

revealed a significant two-way interaction between encoding task and response option, $F(3,195)=142.15, M S E=0.017, p<.001$, such that response rates along the $1-4$ scale differed between the two encoding tasks. Notably, this two-way interaction remained significant when analyzed separately for each group: younger adults, $F(3,138)=108.7$, $M S E=0.014, p<.001$; older adults, $F(3,57)=47.83, M S E=0.022, p<.001$.

Additionally, this mixed effects ANOVA revealed a significant three-way interaction between group, encoding task, and response option, $F(3,195)=4.01, M S E=0.017, p=$ .009 . The interaction arose due to a significant two-way interaction for age group and response option in the distinctiveness task, $F(3,195)=10.40, M S E=0.019, p<.001$, but no age group by response option interaction in the similarity task, $p>.4$. Specifically, in the distinctiveness task, younger adults more frequently used response options in the middle of the scale than at the ends of the scale, such that tests of a quadratic contrast but not a linear contrast were significant, $t(46)=11.74, p<.001$ and $t(46)=0.50, p>.1$. Among older adults, however, tests of quadratic and linear contrasts were both significant, $t(19)=3.02$, $p<.01$ and $t(19)=4.29, p<.001$. When rates of responding in the distinctiveness task were compared directly across groups, analyses revealed that younger adults more frequently assigned faces to the lower end of the scale than did older adults (option 1: $t(65)=$ $2.50, p=.015$; option $2: t(65)=5.05, p<.001)$, and older adults more frequently assigned faces to the upper end of the scale than did younger adults (option $4: t(65)=3.06, p=.003$ ).

\section{Effects of age and learning goals on memory}

To test for possible age-related differences in the effect of encoding strategy on episodic recollection, we conducted a mixed effects ANOVA with age as a between groups factor, and encoding task (distinctiveness, similarity) and quality of memory (remember, know) as repeated measures factors. A significant main effect of encoding task was found, $F(1$, $66)=8.30, M S E=0.005, p=.005$, such that, collapsed across age group and memory quality, hit rates were higher for faces studied in the distinctiveness task than in the similarity task. Results also demonstrated a significant two-way interaction between age group and encoding task, $F(1,66)=9.89, M S E=0.005, p=.003$ (see Figure 3), such that younger adults had higher hit rates for faces studied in the distinctiveness task than in the similarity task, $t(46)=3.95, p<.001$, but older adults showed no difference in hit rates for 


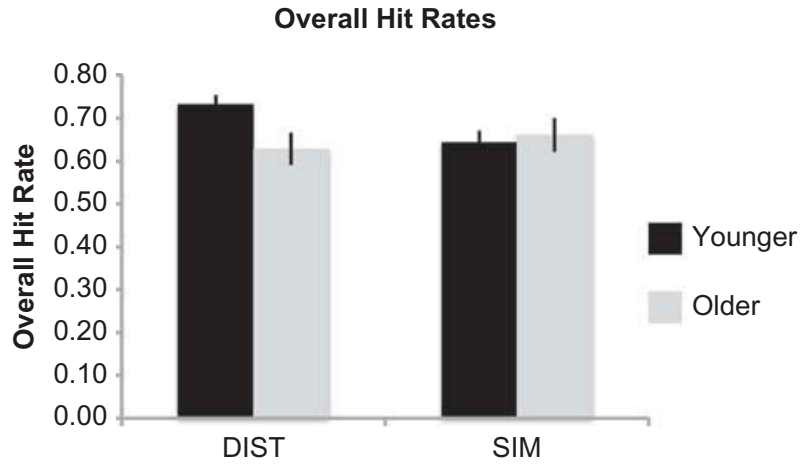

Figure 3. Overall hit rates, collapsed across memory quality, for each age group and each encoding task. Error bars represent standard error of the mean. DIST = distinctiveness task, SIM = similarity task.

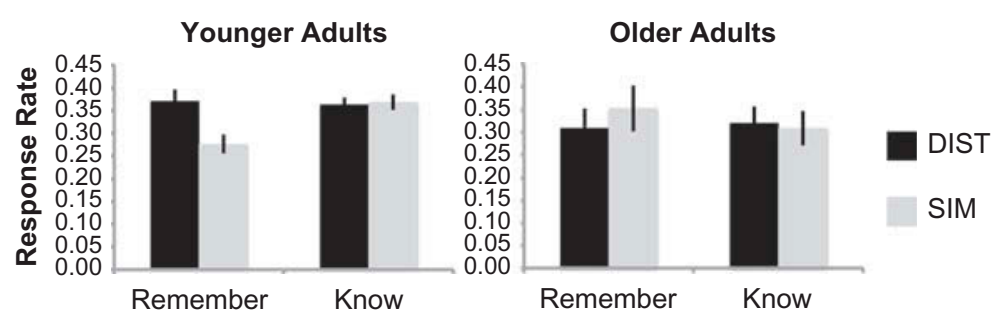

Figure 4. Rates of recollection and familiarity for each age group and each encoding task. Error bars represent standard error of the mean. DIST $=$ distinctiveness task, SIM $=$ similarity task.

faces studied in the two tasks, $p>$.2. With respect to this same interaction, results further showed that younger adults had higher hit rates than older adults for faces studied in the distinctiveness task, $t(46)=2.64, p=.011$, but not in the similarity task, $p>.7$.

Critically, this age group by encoding task interaction was driven primarily by group differences in the rate of recollection for faces studied in each task, as revealed by a significant three-way interaction between age group, encoding task, and memory quality, $F(1,66)=5.38, M S E=0.016, p=.024$ (see Figure 4). Specifically, a significant age group by encoding task interaction was found for remember responses, $F(1,66)=11.11$, $M S E=0.012, p=.001$, but not for known responses, $p>.6$. Post-hoc analyses in younger adults revealed higher rates of recollection following the distinctiveness task than the similarity task, $t(46)=4.00, p<.001$, whereas results in older adults showed that rates of recollection were numerically but not significantly greater for the similarity task than for the distinctiveness task, $p>$.1. Finally, no main effects of age $(p>.2)$ or memory quality $(p>3)$ were found, nor were any interactions found between age group and memory quality $(p>.3)$ or encoding task and memory quality $(p>.1)$.

\section{Relation between encoding ratings and memory}

To further explore the relation between age, encoding task, and memory, we next examined the extent to which distinctiveness and similarity ratings at encoding related 

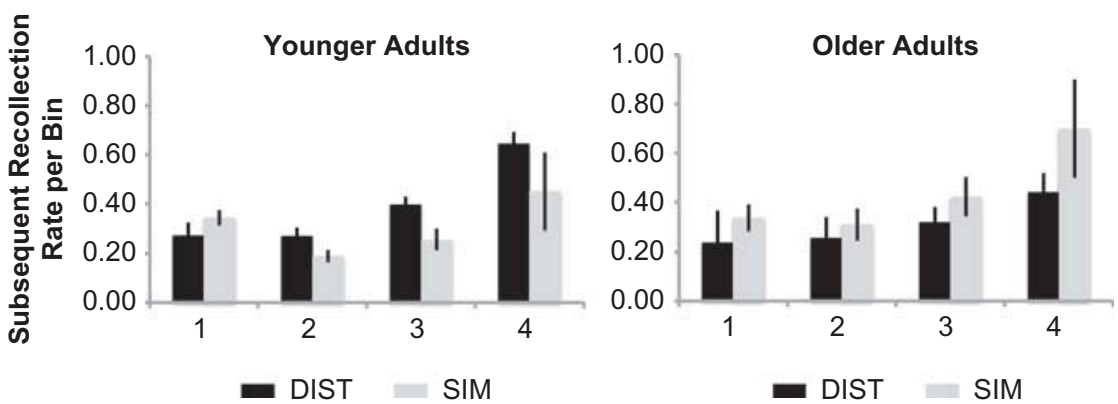

Figure 5. Relationship between encoding task ratings and rates of recollection for each group and each encoding task. Error bars represent standard error of the mean. DIST $=$ distinctiveness task, $\mathrm{SIM}=$ similarity task.

to memory performance in each age group. For each group and each encoding task, faces were binned according to rating and then further subdivided into subsequent remember, know, and miss trials. As such, the proportion of recollected faces for each rating bin could be calculated. Given that not all participants used each rating option during encoding - leading to an unbalanced data set - linear mixed modeling was used to evaluate the relation between encoding task, task ratings, and rates of recollection in each group. Specifically, this analysis tested whether increasing rates of recollection were found with increasing distinctiveness or similarity ratings in each group. In younger adults, results demonstrated a main effect of rating, $X^{2}(1)=13.15, p<.001$, as well as a task by rating interaction, $X^{2}(1)=14.52, p<.001$, such that a linear effect was found for the distinctiveness task, $X^{2}(1)=37.23, p<.001$, but not for the similarity task, $p>.5$ (Figure 5). That is, faces judged as more distinctive at encoding were associated with higher rates of recollection, whereas increasing similarity ratings at encoding were not associated with higher rates of recollection. In contrast, results in older adults demonstrated a main effect of rating, $X^{2}(1)=6.99, p=.008$, but no task by rating interaction, $p>$ .9 (Figure 5), such that increasing distinctiveness and similarity ratings at encoding were both associated with higher rates of recollection.

\section{Effects of age on false memory}

Turning next to false recognition rates, we conducted a mixed effects ANOVA with age group and memory quality (remember or know false alarms) as factors. Note that encoding task was not considered as a third factor due to the nature of the study design - faces from both tasks were intermixed within a single recognition test. Results revealed a significant main effect of age group, $F(1,66)=22.44, M S E=$ $0.007, p<.001$, such that older adults exhibited higher false alarms rates than younger adults, and a significant main effect of memory quality, $F(1,66)=27.18, M S E=0.004$, $p<.001$, such that, collapsed across groups, participants showed higher levels of know false alarms than remember false alarms (Figure 6). A trend for an interaction between age group and memory quality was found, $F(1,66)=3.61, M S E=0.004, p=.062$, with the difference in false alarm rates between younger and older adults slightly greater for remember than know false alarms. 


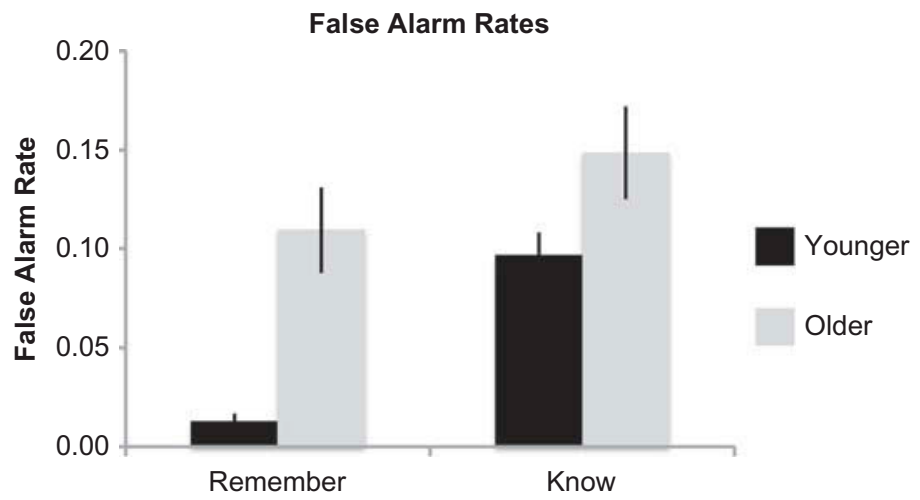

Figure 6. Rates of false recollection and familiarity for each group. Error bars represent standard error of the mean.

\section{Discussion}

The present study examined age-related differences in the manner in which learning strategies encouraging distinctiveness or similarity processing influence recognition memory. Based on prior research, we hypothesized that younger adults would demonstrate enhanced rates of recollection following attention to distinctiveness relative to similarity at encoding, whereas older adults would show equivalent rates of recollection for the two tasks. Results demonstrated that, despite the use of an encoding strategy that explicitly oriented participants to distinctiveness, an age-related reduction in the beneficial effects of distinctiveness was present.

\section{Group differences in encoding distinctiveness}

Analyses of response patterns during the encoding phase of the experiment revealed a significant interaction between encoding task and response option suggesting that, as intended, participants processed faces differently when performing the distinctiveness and similarity tasks. However, analyses further revealed a three-way interaction between age group, encoding task, and response option, such that ratings patterns differed between groups for the distinctiveness task but not for the similarity task. Specifically, younger adults mostly used distinctiveness ratings in the middle of the scale such that responses followed a quadratic trend, whereas patterns of responding among older adults showed both a linear and quadratic trend. Given prior reports of reduced distinctiveness processing in older adults (Bruce \& Herman, 1986; Cimbalo \& Brink, 1982; Mäntylä \& Bäckman, 1990; Rabinowitz et al., 1982; Stark et al., 2013; Toner et al., 2009), one might predict that older adults would rate faces as less distinctive than younger adults. Interestingly, the opposite pattern was found such that older adults assigned faces to the upper end of the distinctiveness scale more frequently than younger adults. These results are not necessarily at odds with prior reports of agerelated impairments in distinctiveness processing, however, in that the lowered distinctiveness threshold observed in older adults may be indicative of decreased processing of distinctive facial attributes. 


\section{Beneficial effects of attending to distinctiveness}

Results from the recognition test revealed a two-way interaction between age group and encoding task such that, collapsed across memory quality, overall hit rates in younger adults were higher for the distinctiveness task than for the similarity task, whereas memory performance did not differ by task in older adults. Specifically, this interaction was driven by group differences in rates of recollection for each task, whereby attending to distinctiveness selectively enhanced recollection but not familiarity in younger adults, but older adults demonstrated no significant task-related differences in rates of recollection. The ability of younger adults to effectively employ distinctiveness processing to their benefit replicates findings by Mäntylä (1997) and more recently by Carr et al. (2013) demonstrating enhanced levels of recollection following encoding that orients attention to distinctive facial features versus similarities among faces.

With respect to older adults, we hypothesized that they would not show the same beneficial boost to recollection as younger adults following distinctiveness encoding based on data suggesting a reduced ability to associate stimuli with their distinctive stimulus features (Naveh-Benjamin, 2000) as well as a reduced ability to encode unique memory representations (Stark et al., 2013; Toner et al., 2009; Wilson et al., 2006). Indeed, older adults exhibited no differences in overall hit rate for faces studied under the two tasks, nor any differences in rates of recollection or familiarity. Older adults also demonstrated higher rates of false recognition than younger adults, lending further support to the notion that older adults may have a diminished capacity to encode new stimuli as distinct from previously studied stimuli (Stark et al., 2013; Toner et al., 2009; Wilson et al., 2006).

These results stand in contrast, to some degree, to results from studies using the isolation effect paradigm, in which older adults are able to overcome deficits in memory for the isolate by making distinctiveness more apparent (e.g., Smith, 2011). Although in the present study older adults were instructed to attend to distinctive facial features, the conditions differed from those used in isolation studies in several ways. First, numerous faces could be considered distinctive from typical faces in many different ways, rather than a single isolated item or a small number of items that differed from their context. Thus, in the current study, the ability of distinctiveness to benefit memory may depend on the ability to acquire multiple unique memory traces, a condition that may not be as critical in the isolation effect. Second, in the isolation paradigm, when participants are able to encode similarities between context items and how these items differ from the isolate, they are able to better specify retrieval cues for the isolates that can constrain memory search and reduce false alarms (Hunt, 2013). In the paradigm used here, there are multiple items that are distinctive in different ways, and thus distinctiveness may not necessarily provide a constraint on retrieval. Finally, based on the framework described by Schmidt (1991), isolation effects tap into effects of primary distinctiveness, in that the isolate is incongruent with other items in the study context. The current paradigm was designed to assess the effects of secondary distinctiveness, in that faces were judged to be distinctive compared to participants' previous experience with faces. Instructions to judge how likely a given face could be picked out of a crowd at the airport would seem to rely on participants comparing the experimental faces to their concept of typical faces in long term memory.

Interestingly, although older adults demonstrated a lower overall hit rate in the distinctiveness task relative to younger adults, performance did not differ between groups for the similarity task. Although results only reached trend levels of significance, older 
adults exhibited a numerically greater rate of recollection for faces studied in the similarity task than did younger adults, $t(46)=1.69, p=.097$. Furthermore, older adults exhibited a numerically greater rate of recollection for faces studied in the similarity task relative to those studied in the distinctiveness task. An interesting question for further study is whether encoding processes that highlight attention to similarity may strengthen gistbased memory representations in older adults, and in turn lead to preserved memory performance. It also may be that older adults have a "default mode" of using gist-based encoding (Brainerd et al., 2009; Koutstaal \& Schacter, 1997), and can thus benefit when attending to similarities among encoded stimuli. Providing initial support for this notion, analyses of the relation between task ratings at encoding and memory performance revealed that in older but not in younger adults, increasing similarity ratings were associated with increasing rates of recollection. An alternate interpretation of the similarity data, however, is that rather than benefit memory performance in older adults, attending to similarity reduces memory performance in younger adults such that the two groups show equivalent rates of recollection.

A potential caveat to the current findings is that participants from both age groups were presented with photographs of young adults. Although literature exists which suggests an own-age bias in recognition memory for faces (Anastasi \& Rhodes, 2005), other data exist which demonstrate an own-age bias for younger, but not older adults (Lindholm, 2005). Although it is possible that memory performance in older adults would have improved had they studied photographs of older adults, it is unlikely that an own-age bias can account for the current pattern of findings. First, older adults rated the faces as more distinctive than younger adults did, so it was not the case that older adults were insensitive to the uniqueness of young adult faces. Second, older adults recognized the young adult faces presented in the similarity task as well as younger adults did. The only difference found between the two age groups was a specific enhancement of recollection levels for faces studied in the distinctiveness task in younger but not older adults, a finding unlikely to arise from the use of young adult faces.

\section{Conclusions}

The present study examined age-related differences in the manner in which task demands during learning affected memory performance. Results demonstrated group differences both in the manner in which participants engaged in the encoding tasks as well as in how these tasks affected memory performance. Critically, whereas attending to distinctiveness relative to similarity benefited rates of recollection in younger adults, recollection performance did not differ by task in older adults. The vast majority of previous studies examining age-related changes in distinctiveness processing have assessed memory performance following the use of spontaneous or nonoriented encoding strategies. The present study adds to this body of literature by demonstrating that, despite the use of an encoding strategy that explicitly oriented participants to distinctiveness, an age-related reduction in the beneficial effects of distinctiveness on episodic memory was still found. Interestingly, recollection in older adults may benefit to some extent from an encoding strategy that strengthens gist-based memory. These results suggest that attending to similarities across items may be an effective encoding strategy in older adults, though further research is needed to better understand the conditions under which older adults may benefit from encoding and retrieval operations based on distinctiveness and similarity. 


\section{Funding}

This work was supported by the National Science Foundation [grant number BCS 0848246] awarded to Barbara J. Knowlton, Achievement Rewards for College Scientists awarded to Valerie A. Carr, and the University of California Los Angeles Dissertation Year Fellowship awarded to Valerie A. Carr.

\section{References}

Anastasi, J. S., \& Rhodes, M. G. (2005). An own-age bias in face recognition for children and older adults. Psychonomic Bulletin \& Review, 12, 1043-1047. doi:10.3758/BF03206441

Badham, S. P., \& Maylor, E. A. (2013). Age-related associative deficits and the isolation effect. Aging, Neuropsychology, and Cognition, 20, 405-428. doi:10.1080/13825585.2012.725460

Badham, S. P., Wade, K. A., Watts, H. J., Woods, N. G., \& Maylor, E. A. (2013). Replicating distinctive facial features in lineups: Identification performance in young versus older adults. Psychonomic Bulletin \& Review, 20, 289-295. doi:10.3758/s13423-012-0339-2

Bireta, T. J., Surprenant, A. M., \& Neath, I. (2008). Age-related differences in the Von Restorff isolation effect. The Quarterly Journal of Experimental Psychology, 61, 345-352. doi:10.1080/ 17470210701626608

Brainerd, C. J., Reyna, V. F., \& Howe, M. L. (2009). Trichotomous processes in early memory development, aging, and neurocognitive impairment: A unified theory. Psychological Review, 116, 783-832. doi:10.1037/a0016963

Bruce, P. R., \& Herman, J. F. (1986). Adult age differences in spatial memory: Effects of distinctiveness and repeated experience. Journal of Gerontology, 41, 774-777. doi:10.1093/geronj/ 41.6.774

Carr, V. A., Engel, S. A., \& Knowlton, B. J. (2013). Top-down modulation of hippocampal encoding activity as measured by high-resolution functional MRI. Neuropsychologia, 51, 1829-1837. doi:10.1016/j.neuropsychologia.2013.06.026

Chun, M. M., \& Turk-Browne, N. B. (2007). Interactions between attention and memory. Current Opinion in Neurobiology, 17, 177-184. doi:10.1016/j.conb.2007.03.005

Cimbalo, R. S., \& Brink, L. (1982). Aging and the Von Restorff isolation effect in short/term memory. The Journal of General Psychology, 106(1st Half), 69-76. doi:10.1080/ 00221309.1982 .9710974

Craik, F. I. M. (2001). Effects of dividing attention on encoding and retrieval processes. In H. L. Roediger (Ed.), The nature of remembering: Essays in honor of Robert G. Crowder (pp. 55-68). New York, NY: American Psychological Association.

Deese, J. (1959). On the prediction of occurrence of particular verbal intrusions in immediate recall. Journal of Experimental Psychology, 58, 17-22. doi:10.1037/h0046671

Dodson, C. S., \& Schacter, D. L. (2002). Aging and strategic retrieval processes: Reducing false memories with a distinctiveness heuristic. Psychology and Aging, 17, 405-415. doi:10.1037/ 0882-7974.17.3.405

Eldridge, L. L., Sarfatti, S., \& Knowlton, B. J. (2002). The effect of testing procedure on rememberknow judgments. Psychonomic Bulletin \& Review, 9, 139-145. doi:10.3758/BF03196270

Folstein, M. F., Folstein, S. E., \& McHugh, P. R. (1975). "Mini-mental state". A practical method for grading the cognitive state of patients for the clinician. Journal of Psychiatric Research, 12, 189-198. doi:10.1016/0022-3956(75)90026-6

Gallo, D. A., \& Roediger III, H. L. (2003). The effects of associations and aging on illusory recollection. Memory \& Cognition, 31, 1036-1044. doi:10.3758/BF03196124

Geraci, L., McDaniel, M. A., Manzano, I., \& Roediger III, H. L. (2009). The influence of age on memory for distinctive events. Memory \& Cognition, 37, 175-180. doi:10.3758/ MC.37.2.175

Hunt, R. R. (2006). The concept of distinctiveness in memory research. In R. R. Hunt \& J. B. Worthen (Eds.), Distinctiveness and memory (pp. 3-25). Oxford: Oxford University Press.

Hunt, R. R. (2013). Precision in memory through distinctive processing. Current Directions in Psychological Science, 22, 10-15. doi:10.1177/0963721412463228

Koutstaal, W., \& Schacter, D. L. (1997). Gist-based false recognition of pictures in older and younger adults. Journal of Memory and Language, 37, 555-583. doi:10.1006/ jmla.1997.2529 
Lin, O. Y., \& MacLeod, C. M. (2012). Aging and the production effect: A test of the distinctiveness account. Canadian Journal of Experimental Psychology/Revue Canadienne De Psychologie Expérimentale, 66, 212-216. doi:10.1037/a0028309

Lindholm, T. (2005). Own-age biases in verbal person memory. Memory, 13, 21-30. doi:10.1080/ 09658210344000549

Logan, J. M., Sanders, A. L., Snyder, A. Z., Morris, J. C., \& Buckner, R. L. (2002). Underrecruitment and nonselective recruitment: Dissociable neural mechanisms associated with aging. Neuron, 33, 827-840. doi:10.1016/S0896-6273(02)00612-8

Mäntylä, T. (1993). Knowing but not remembering: Adult age differences in recollective experience. Memory \& Cognition, 21, 379-388. doi:10.3758/BF03208271

Mäntylä, T. (1997). Recollections of faces: Remembering differences and knowing similarities. Journal of Experimental Psychology: Learning, Memory, and Cognition, 23, 1203-1216. doi:10.1037/0278-7393.23.5.1203

Mäntylä, T., \& Bäckman, L. (1990). Encoding variability and age-related retrieval failures. Psychology and Aging, 5, 545-550. doi:10.1037/0882-7974.5.4.545

McDaniel, M. A., Einstein, G. O., \& Jacoby, L. L. (2008). New considerations in aging and memory: The glass may be half full. In F. I. M. Craik \& T. Salthouse (Eds.), The handbook of aging and cognition (3rd ed., pp. 255-310). Hove: Psychology Press.

Naveh-Benjamin, M. (2000). Adult age differences in memory performance: Tests of an associative deficit hypothesis. Journal of Experimental Psychology: Learning, Memory, and Cognition, 26, 1170-1187. doi:10.1037/0278-7393.26.5.1170

Old, S. R., \& Naveh-Benjamin, M. (2008). Differential effects of age on item and associative measures of memory: A meta-analysis. Psychology and Aging, 23, 104-118. doi:10.1037/08827974.23.1.104

Perfect, T. J., Williams, R. B., \& Anderton-Brown, C. (1995). Age differences in reported recollective experience are due to encoding effects, not response bias. Memory, 3, 169-186. doi:10.1080/09658219508258964

Prull, M. W., Dawes, L. L., Martin III, A. M., Rosenberg, H. F., \& Light, L. L. (2006). Recollection and familiarity in recognition memory: Adult age differences and neuropsychological test correlates. Psychology and Aging, 21, 107-118. doi:10.1037/0882-7974.21.1.107

Rabinowitz, J. C., Craik, F. I., \& Ackerman, B. P. (1982). A processing resource account of age differences in recall. Canadian Journal of Psychology/Revue Canadienne De Psychologie, 36, 325-344. doi:10.1037/h0080643

Roediger III, H. L., \& McDermott, K. B. (1995). Creating false memories: Remembering words not presented in lists. Journal of Experimental Psychology: Learning, Memory, and Cognition, 21, 803-814. doi:10.1037/0278-7393.21.4.803

Schacter, D. L., Israel, L., \& Racine, C. (1999). Suppressing false recognition in younger and older adults: The distinctiveness heuristic. Journal of Memory and Language, 40, 1-24. doi:10.1006/ jmla.1998.2611

Schmidt, S. R. (1991). Can we have a distinctive theory of memory? Memory \& Cognition, 19, 523542. doi:10.3758/BF03197149

Smith, R. E. (2006). Adult age differences in episodic memory: Item-specific, relational, and distinctive processing. In R. R. Hunt \& J. B. Worthen (Eds.), Distinctiveness and memory (pp. 259-287). Oxford: Oxford University Press.

Smith, R. E. (2011). Providing support for distinctive processing: The isolation effect in young and older adults. Psychology and Aging, 26, 744-751. doi:10.1037/a0022715

Smith, R. E., \& Hunt, R. R. (1998). Presentation modality affects false memory. Psychonomic Bulletin \& Review, 5, 710-715. doi:10.3758/BF03208850

Smith, R. E., Lozito, J. P., \& Bayen, U. J. (2005). Adult age differences in distinctive processing: The modality effect on false recall. Psychology and Aging, 20, 486-492. doi:10.1037/08827974.20.3.486

Stark, S. M., Yassa, M. A., Lacy, J. W., \& Stark, C. E. (2013). A task to assess behavioral pattern separation (BPS) in humans: Data from healthy aging and mild cognitive impairment. Neuropsychologia, 51, 2442-2449. doi:10.1016/j.neuropsychologia.2012.12.014

Toner, C. K., Pirogovsky, E., Kirwan, C. B., \& Gilbert, P. E. (2009). Visual object pattern separation deficits in nondemented older adults. Learning \& Memory, 16, 338-342. doi:10.1101/ Im.1315109 
Tulving, E. (1985). Memory and consciousness. Canadian Psychology/Psychologie Canadienne, 26, 1-12. doi: $10.1037 / \mathrm{h} 0080017$

Vitali, P., Minati, L., Chiarenza, G., Brugnolo, A., Girtler, N., Nobili, F., ... Rodriguez, G. (2006). The Von Restorff effect in ageing and Alzheimer's disease. Neurological Sciences, 27, 166-172. doi:10.1007/s10072-006-0662-3

Wilson, I. A., Gallagher, M., Eichenbaum, H., \& Tanila, H. (2006). Neurocognitive aging: Prior memories hinder new hippocampal encoding. Trends in Neurosciences, 29, 662-670. doi:10.1016/j.tins.2006.10.002 